\title{
Health Outcomes and Cost Considerations of Assisted Peritoneal Dialysis: A Narrative Review
}

\author{
Serban M. Maierean ${ }^{a}$ Matthew J. Oliver ${ }^{a, b}$ \\ a'Department of Medicine, University of Toronto, Toronto, ON, Canada; ${ }^{b}$ Division of Nephrology, \\ Sunnybrook Health Sciences Centre, Toronto, ON, Canada
}

\section{Keywords}

Assisted peritoneal dialysis · Hemodialysis · Quality of life · Mortality $\cdot$ Cost $\cdot$ Barriers $\cdot$ Outcomes

\begin{abstract}
Background: Peritoneal dialysis (PD) is underutilized in many parts of the world despite pro-PD health policies. The physical and cognitive demands of PD means that over half of eligible patients require some form of assistance. As such, many countries now offer assisted PD (aPD) programs to help patients start or stay on PD as opposed to in-center hemodialysis (HD). In order to evaluate the potential scope of aPD, it is important to review the outcomes and cost considerations of aPD. Summary: We reviewed available data from different countries and regions for health outcomes between aPD and in-center HD, with a focus on quality of life (QoL), mortality, hospitalization, and technique survival. We also evaluated studies discussing the overall costs of delivering $\mathrm{aPD}$, including training, operating costs, and indirect costs and compared these to in-center HD costs for the same regions. Key Messages: aPD patients are older and more frail than either self-care PD patients and many in-center HD patients. We found no evidence for any difference in QoL, mortality, or hospitalization between aPD and in-center HD after
\end{abstract}

adjustment for these differences. There is some evidence for an association between nurse assistance and improved technique survival as compared to family assistance or selfcare PD. Despite increased cost of providing assistance in PD, it is still significantly less expensive than in-center HD in Western Europe and Canada.

(c) 2021 The Author(s)

Published by S. Karger AG, Basel

\section{Introduction}

Peritoneal dialysis (PD) is a significantly underutilized renal replacement therapy (RRT) in many regions. As compared to in-center hemodialysis (HD), self-care PD boasts at least similar medical outcomes [1], improved physical health-related quality of life (QoL) [2], and increased satisfaction with their treatment [3]. Self-care PD is also more cost-effective than in-center HD [4], and in aggregate, consensus among surveyed nephrologists is that at least $40 \%$ of patients requiring RRT should be on some form of PD [3]. Despite this, of all incident ESRD patients, PD is used in only $10.1 \%$ (USA) [5], 11\% (Europe) [6], and 22.4\% (Canada) [7] of cases. Multiple barriers stand in the way of $\mathrm{PD}$ uptake, especially in managing one's own $\mathrm{PD}$, which requires multiple skills that de- 
pend on good physical and cognitive functioning. In one Canadian cohort consisting of 121 patients, aged $69 \pm 10$ years, only $5 \%$ of subjects were fully independent [8]. Sixty two percent required some assistance in managing their PD, of which $34 \%$ came from friends/family and $28 \%$ from a paid caregiver [8]. This is corroborated in a Saudi cohort where $49.5 \%$ of patients required at least some assistance with their dialysis [9]. As a patient's frailty and cognition deteriorates, the demands placed on the family necessarily increase, which can become overwhelming. Many patients fear they will not be able to manage, and this itself can serve as a significant barrier to using PD [10].

In order to overcome these patient-related barriers, some countries have implemented assisted PD (aPD) programs where a nurse visits the patient at home, often multiple times a day, to assist them with their dialysis. In France, $50.1 \%$ of patients on PD receive some assistance with their dialysis, and of these, $82 \%$ are assisted primarily by a trained nurse [11]. Despite having a robust aPD program and the fact that $45 \%$ of patients would choose $\mathrm{PD}$ as their RRT when provided with equal information about all RRTs [12], only $\sim 10 \%$ of incident dialysis patients in France are started on PD [6] but with large regional variability (2.9-26.5\%) [12]. Durand et al. [12] discuss key factors contributing to this gap, including understaffing, limited PD training for new nephrologists, limited information about PD provided to patients requiring dialysis, and lack of financial incentive.

In order to help promote tackling these barriers, it is important to understand whether aPD confers any advantages over in-center HD. As such, the focus of this review is to compare aPD to in-center $\mathrm{HD}$ by evaluating health outcome differences as well as cost differences in diverse areas of the world. We will review studies that compare QoL, mortality, and technique survival between aPD and in-center HD, and to look at cost data in order to provide a meaningful view of the merits of $\mathrm{aPD}$.

\section{Assisted Peritoneal Dialysis}

With an increasing aging population seen in most Western countries comes a higher incidence of ESRD and dependence on RRT. While many areas around the world have implemented PD-first (Hong Kong and Thailand) and PD-favored (Canada, USA, Spain, India, China, Mexico, and Guatemala) policies [13], PD is still underutilized, particularly in the latter countries and especially among the elderly population. aPD aims to ad- dress the increasing difficulties in managing one's own $\mathrm{PD}$ as a significant portion of this patient population suffers from physical and/or cognitive impairments. In order to better understand the role of $\mathrm{aPD}$, a study from Toronto, Canada, explored home dialysis patients' needs by studying 121 patients aged $50+$ (mean $69 \pm 10$ years) across 3 dialysis sites [8]. They looked at the different tasks associated with managing ones own $\mathrm{PD}$, including instrumental tasks (i.e., organizing clinic visits, medications, and supplies) and basic tasks (i.e., setting up the cycler [if applicable], documenting, troubleshooting, connecting, lifting and discarding bags, exist-site care, and assessment of dialysis effectiveness) [8]. In every area, many patients required family or nurse assistance. Overall, $62 \%$ of patients required at least some help with their PD, of which $23 \%$ required the help from both family and a nurse. Patients who needed help required assistance with, on average, 3 basic and 2 instrumental tasks [8]. Although this study only looked at an older patient population, these findings are corroborated in France, where the French PD registry (RDPFL) found that $56 \%$ of its patients were not able to perform PD unassisted [14].

When family and/or nursing assistance is available and easy to access, it is commonly used. The observed effect of this is the older average age of patients on PD in countries where aPD is offered. For example, in Germany patients with ESRD started on PD are on average 60 years of age (62.5 male/57.0 female), compared to HD where they are 72 years of age (71.0 male/73.0 female) [15]. Meanwhile in France, where there exists a well-funded aPD program, the median age of patients on PD is 71 years [14]. PD uptake improved with a HR of 1.13 (95\% CI 1.05-1.21) for increased rate of CAPD utilization for nurse-assisted PD patients [16]. This relationship is corroborated in another study looking at 134 incident ESRD patients in Ontario, $80 \%$ of whom had at least 1 personal barrier to self-care PD [17].

aPD programs overcome many of the barriers that patients face in managing their own care. Through these programs, patients who were previously ineligible for $\mathrm{PD}$ would have access to home dialysis, and this is particularly impactful to more elderly patients.

\section{Health Outcomes on aPD}

Patients requiring assistance for $\mathrm{PD}$ are older and have more comorbidities than those on self-care PD and many of those on in-center HD [18, 19]. As such, data regarding 
health outcomes between these groups need to account for these differences in order to understand whether maintaining patients on PD with assistance is of benefit.

\section{Quality of Life}

For home dialysis in general, a systematic review and meta-analysis looking at 46 publications, pooling together 4,158 home dialysis and 7,854 in-centre HD patients from 40 distinct patient populations was conducted [2]. They found no overall difference in either physical or mental health-related QoL (that is, a patient's perception of one's own physical or mental well-being, respectively). They also noted a high degree of bias among many of the studies they included. Another study looking at aPD specifically, which was not included in the meta-analysis due to differences in scoring systems, matched $106 \mathrm{aPD}$ patients to $100 \mathrm{HD}$ patients from the UK and Northern Ireland, and evaluated them using the Hospital Anxiety and Depression Scale (HADS), SF-12 physical and mental scores, Renal Treatment Satisfaction Questionnaire (RTSQ), symptom score, Barthel's score, and Illness Intrusiveness Rating Scale (IIRS). Similarly, no significant differences were seen between $\mathrm{APD}$ and HD patients with respect to any score [20].

\section{Mortality and Hospitalization}

A large retrospective multicentre cohort from Toronto, Canada, comparing aPD $(n=203)$ with in-patient HD $(n=872)$ (propensity score-adjusted for baseline differences) found no differences in overall mortality or overall days spent in hospital between the 2 groups [19]. Wakeel et al. [9] also found no difference in mortality between aPD and HD groups, but cohorts were not matched. More data are needed to definitively show similar mortality rates.

\section{Technique Failure}

Among those receiving some form of assistance with their PD, a study based on the French RDPFL found lower rates of technique failure than non-assisted patients (HR 0.85, 95\% CI 0.77-0.94) [18]. They also demonstrated the importance of PD center volumes, finding a lower risk of technique failure among centers with 10-20 and $20+$ new patients per year as compared to centers with fewer than 10 patients per year (adjusted cause-specific HR 0.84 [95\% CI 0.77-0.93] and 0.83 [95\% CI 0.72-0.94], respectively) [18]. Another study based on the RDPFL found similar rates of peritonitis between assisted and non-assisted patients, but a lower risk of transfer to HD for patients assisted by nurses as opposed to family alone
(HR 0.85, 95\% CI 0.75-0.97) [11]. These findings on peritonitis risk are contradicted by another study looking at 1,953 incident dialysis patients on CAPD, which found that the patients requiring assistance by a health-care provider had significantly higher rates of peritonitis than patients who undergo self-care $\mathrm{PD}(\mathrm{HR}=2.64,95 \% \mathrm{CI}=$ $1.23-5.64, p=0.012$ ) [21]. Interestingly, while overall age $>65$ was positively correlated with risk of peritonitis as compared to those younger than 65 , this relationship only held for patients in the aPD cohort (HR $=2.09,95 \% \mathrm{CI}$ $1.33-3.27,0.001)$ and not for those able to undergo selfcare $\mathrm{PD}(\mathrm{HR}=0.87,95 \% \mathrm{CI}=0.50-1.52, p=0.62)$ [21]. However, the authors did not control for comorbidities in the aPD groups aged $65+$ and $<65$, and comparison groups between assisted and self-care PD were not age- or comorbidity-matched. A recent systematic review looking at health outcomes of different aPD models around the world found overall evidence surrounding peritonitis to be heterogeneous, but with overall no evidence of a difference between various levels of assistance and self-care $\mathrm{PD}$ with respect to peritonitis or overall technique survival [22].

Overall, current evidence suggests no difference in QoL between aPD and in-center HD. Moreover, we found no evidence of a difference in mortality or hospitalization between aPD and in-center HD. There is conflicting evidence evaluating whether patients requiring assistance with PD experience similar rates of peritonitis and technique survival as those on self-care PD. More research is required to address the aforementioned.

\section{Cost Considerations of aPD}

When implementing an aPD system, it is important to also keep in mind the multiple factors involved in ensuring the delivery of high quality care. Competence in $\mathrm{aPD}$ is a process that can require up to $20 \mathrm{~h}$ of training for a nurse and requires specialized certification in some countries [23]. Patients' needs are then assessed by a visiting nurse who observes the patient perform an entire PD session [23], and this might be repeated multiple times throughout a patient's life as they require more assistance. Staffing requirements should also be clearly accounted for and be somewhat flexible, as patients' needs can vary widely and do change over time. One study from Canada found patients on APD which will require just under 7 visits per week [17].

In Western Europe, the added cost of providing assistance to patients on PD varies widely [23]. In France, for 
example, the yearly average per-patient cost for providing assistance to patients on PD, including nurse salary, transportation, and training, is around 23,400 Euro for CAPD and 18,200 Euro for APD. Whereas in Belgium, it is only 9,360 Euro for CAPD and 5,356 Euro for APD (which covers up to 2 daily home visits). Despite the high cost of assistance in France, the total cost of in-centre HD is still higher [23] than the total cost PD with assistance, a figure which does not even include the initial cost of building and installing the HD facility [24].

In Ontario, a comprehensive per-patient cost for patients on PD (including both self- and aPD) is $\$ 86,211$ $(95 \%$ CI $83,832-88,257)$ for the first year, and $\$ 166,777$ $(162,540-171,862)$ for the first 2 years combined. By comparison, the total per-patient cost of in-center HD (excluding patient travel costs) is $\$ 141,982$ (95\% CI 139,774$144,361)$ for the first year and $\$ 234,824(231,596-238,489)$ for the first 2 years combined [4]. Estimates from Ontario regarding the cost of providing an average of one nursing visit daily to a PD patient is approximately $\$ 18,534$ per year including all care, travel, training, and administrative costs [25]. Looking at the difference in total costs and the cost of providing PD assistance, in Ontario, demonstrates the cost-effectiveness of aPD in this region. In Alberta, the total net cost of in-centre HD is about $\$ 23,500$ more per patient, per year than aPD [26], while in British Columbia this difference is almost $\$ 30,000$ [25].

About $10 \%$ of patients on PD require nursing assistance only temporarily with periods of 60 days or less (data provided by the Ontario Renal Network). For these patients, some provinces in Canada provide temporary access to aPD, after which they are expected to continue managing PD by themselves or with family assistance. The additional cost of assistance for this group is only $\$ 1,247$ per 30-day period [25].

Cost could be further reduced, for example, by introducing nurses into some of the decision-making process or by increasing use of telemedicine to address some of the cognitive barriers to PD. With regards to the former, one recent cohort found nurses' subjective ability to assess patients' need for assistance correlated strongly with their use of assistance [27]. With regards to the idea of telemedicine, the COVID-19 pandemic has created numerous such opportunities, and one recent study looking at patients on PD described excellent results and patient uptake [28].

Overall, when considering Canada and Western Europe, the cost of aPD is significantly lower than in-center HD. There also exists potential for significant cost reductions related to aPD through avenues like telemedicine and promoting increased nursing autonomy.

\section{Discussion and Conclusion}

PD is significantly underutilized in many regions of the world. Many efforts in the way of PD-first or PD-favored health policies have been made to promote its use [13]. And while some regions like Thailand and Hong Kong, which have strong "PD-first" policies, have significantly increased uptake, most other countries with only "PD-favored" health policies still lag behind [13]. This is largely because many patients who could benefit from PD experience physical and/or cognitive barriers to managing dialysis by themselves [8], and also lack the supports needed to compensate. Indeed, providing home supports to these patients has increased uptake of PD is some areas, like the UK and Canada [16, 17]. However, in other places, like France, it has not [12]. In order to improve PD uptake, governments must identify the relevant patientand institution-related barriers, and work to reduce or eliminate them. In addition, it is important to create a strong assistance program that meets the needs of its population.

With regards to health outcomes, current data show no QoL differences between in-patient HD and aPD [2, 20], with large heterogeneity between studies [2]. Mortality and hospitalization outcome differences are still being debated, though current data show no difference [9] after adjusting for age, gender, and comorbidities [19]. However, both of these studies are based on retrospective data. There is some evidence that providing nursing assistance and higher center volumes are associated with lower rates of technique failure [18]. But overall, it is unclear if rates of peritonitis and technique failure are different between patients on self-care PD and those requiring assistance.

In conclusion, aPD provides many older patients with barriers to self-care PD the opportunity to receive home dialysis. aPD has similar health outcomes and lower costs than in-center HD. Supplementing aPD with telemedicine or offering visiting nurses autonomy, will be very valuable as adoption of aPD increases.

\section{Statement of Ethics}

This study is exempt from Ethical Committee approval as it is a narrative review of previously published material.

\section{Conflict of Interest Statement}

Serban Maierean: none. Matthew Oliver: speaking honorarium from Baxter Healthcare, co-inventor of DMAR systems. 


\section{Funding Sources}

Self-funded.

\section{References}

1 Mehrotra R, Chiu YW, Kalantar-Zadeh K, Bargman J, Vonesh E. Similar outcomes with hemodialysis and peritoneal dialysis in patients with end-stage renal disease. Arch Intern Med. 2011 Jan;171(2):110-8.

2 Bonenkamp AA, van Eck van der Sluijs A, Hoekstra T, Verhaar MC, van Ittersum FJ, Abrahams AC, et al. Health-related quality of life in home dialysis patients compared to incenter hemodialysis patients: a systematic review and meta-analysis. Kidney Med. 2020 Mar;2(2):139-54

3 Sinnakirouchenan R, Holley JL. Peritoneal dialysis versus hemodialysis: risks, benefits, and access issues. Adv Chronic Kidney Dis. 2011 Nov;18(6):428-32.

4 Krahn MD, Bremner KE, de Oliveira C, Dixon SN, McFarlane P, Garg AX, et al. Home dialysis is associated with lower costs and better survival than other modalities: a population-based study in Ontario, Canada. Perit Dial Int. 2019 Dec;39(6):553-61.

5 United States Renal Data System. 2019 USRDS annual data report: epidemiology of kidney disease in the United States.

6 European Renal Association-European Dialysis and Transplant Association. ERA-EDTA Registry Annual Report 2017.

7 Canadian Organ Replacement Register. Treatment of End-Stage Organ Failure in Canada, Canadian Organ Replacement Register, 2009 to 2018: End-Stage Kidney Disease and Kidney Transplants.

8 Farragher JF, Oliver MJ, Jain AK, Flanagan S, Koyle K, Jassal SV. PD assistance and relationship to co-existing geriatric syndromes in incident peritoneal dialysis therapy patients. Perit Dial Int. 2019 Jul;39(4):375-81.

9 Wakeel JSA, Ghonaim MAA, Aldohayan A, Usama S, Obaili SA, Tarakji AR, et al. Appraising the outcome and complications of peritoneal dialysis patients in self-care peritoneal dialysis and assisted peritoneal dialysis: a 5 -year review of a single Saudi center. Saudi J Kidney Dis Transpl. 2018 Jan;29(1):71.

\section{Author Contributions}

Serban Maierean: data collection, manuscript synthesis, and manuscript editing. Matthew Oliver: data collection, manuscript editing, and final approval for publication.
10 Lavoie JG, Zacharias J, Kaufert J, Krueger N, Kinew KA, Mcleod L, et al. Is assisted peritoneal dialysis a solution for northern manitoba? Healthc Policy. 2019;14(4):52-65.

11 Guilloteau S, Lobbedez T, Guillouët S, Verger C, Ficheux M, Lanot A, et al. Impact of assisted peritoneal dialysis modality on outcomes: a cohort study of the French language peritoneal dialysis registry. Am J Nephrol. 2018;48(6):425-33.

12 Durand PY, Verger C. The state of peritoneal dialysis in France. Perit Dial Int. 2006 Nov; 26(6):654-7.

13 Liu FX, Gao X, Inglese G, Chuengsaman P, Pecoits-Filho R, Yu A. A global overview of the impact of peritoneal dialysis first or favored policies: an opinion. Perit Dial Int. 2015;35(4):406-20.

14 Verger C, Ryckelynck JP, Duman M, Veniez G, Lobbedez T, Boulanger E, et al. French peritoneal dialysis registry (RDPLF): outline and main results. Kidney Int Suppl. 2006 Nov(103):S12-20.

15 Pommer W, Su X, Zhang M, Liu F, Yin L. Implementing assisted peritoneal dialysis in renal care: a Chinese-German perspective. Kidney Blood Press Res. 2018;43(5):1646-54.

16 Boyer A, Solis-Trapala I, Tabinor M, Davies SJ, Lambie M. Impact of the implementation of an assisted peritoneal dialysis service on peritoneal dialysis initiation. Nephrol Dial Transplant. 2020 Sep 1;35(9):1595-601.

17 Oliver MJ, Quinn RR, Richardson EP, Kiss AJ, Lamping DL, Manns BJ. Home care assistance and the utilization of peritoneal dialysis. Kidney Int. 2007 Apr;71(7):673-8

18 Lobbedez T, Verger C, Ryckelynck JP, Fabre E, Evans D. Is assisted peritoneal dialysis associated with technique survival when competing events are considered? Clin J Am Soc Nephrol. 2012 Apr;7(4):612-8.

19 Oliver MJ, Al-Jaishi AA, Dixon SN, Perl J, Jain AK, Lavoie SD, et al. Hospitalization rates for patients on assisted peritoneal dialysis compared with in-center hemodialysis. Clin J Am Soc Nephrol. 2016 Sep;11(9):1606-14.
20 Iyasere $\mathrm{O}$, Brown E, Gordon F, Collinson $\mathrm{H}$, Fielding R, Fluck R, et al. Longitudinal trends in quality of life and physical function in frail older dialysis patients: a comparison of assisted peritoneal dialysis and in-center hemodialysis. Perit Dial Int. 2019 Apr;39(2):112-8.

21 Xie D, Zhou J, Cao X, Zhang Q, Sun Y, Tang $\mathrm{L}$, et al. Percutaneous insertion of peritoneal dialysis catheter is a safe and effective technique irrespective of BMI. BMC Nephrol. 2020 May;21(1): 199.

22 Hofmeister M, Klarenbach S, Soril L, ScottDouglas N, Clement F. A systematic review and jurisdictional scan of the evidence characterizing and evaluating assisted peritoneal dialysis models. Clin J Am Soc Nephrol. 2020 Apr;15(4):511-20.

23 Giuliani A, Karopadi AN, Prieto-Velasco M, Manani SM, Crepaldi C, Ronco C. 2017 Sep.

24 Béchade C, Lobbedez T, Ivarsen P, Povlsen JV. Assisted peritoneal dialysis for older people with end-stage renal disease: the French and Danish experience. Perit Dial Int. 2015 Nov;35(6):663-6.

25 Bevilacqua MU, Turnbull L, Saunders S, Er L, Chiu H, Hill P, et al. Evaluation of a 12-month pilot of long-term and temporary assisted peritoneal dialysis. Perit Dial Int. 2017 Jun; 37(3):307-13.

26 Oliver MJ, Salenger P. Making assisted peritoneal dialysis a reality in the United States: a Canadian and American viewpoint. Clin J Am Soc Nephrol. 2020 Apr 7;15:566-8.

27 Guillouët S, Boyer A, Lanot A, Ficheux M, Lobbedez T, Béchade C. Assessment for assisted peritoneal dialysis by peritoneal dialysis nurses: results of a cohort study. Am J Nephrol. 2019;50(6):489-98

28 Scarpioni R, Manini A, Chiappini P. Remote patient monitoring in peritoneal dialysis helps reduce risk of hospitalization during Covid-19 pandemic. J Nephrol. 2020 Dec; 33(6):1123-4. 